\title{
Distribution, incidence, prevalence and default of patients with diabetes mellitus accessing public healthcare in the 11 districts of KwaZulu-Natal, South Africa
}

\author{
N Sahadew, ${ }^{1}$ BTech (Podiatry); V S Singaram, ${ }^{1}$ BMedSc, MMedSc, PhD; S Brown, ${ }^{2}$ MB BCh, FCP (SA), MMed (Med) \\ ${ }^{1}$ Clinical and Professional Practice, School of Clinical Medicine, College of Health Sciences, Nelson R Mandela School of Medicine, \\ University of KwaZulu-Natal, Durban, South Africa \\ ${ }^{2}$ Mahatma Gandhi Memorial Hospital, Durban, South Africa
}

Corresponding author: N Sahadew (nsahadew@gmail.com)

\begin{abstract}
Background. The global increase in the prevalence of diabetes mellitus is most marked in African countries. The District Health Information System (DHIS) is the primary data collection system of the Department of Health in KwaZulu-Natal Province (KZN), South Africa. Data are routinely collected at all public healthcare facilities in the province and are aggregated per facility.

Objective. To investigate the distribution, incidence and prevalence of diabetes in the public healthcare sector of KZN.

Methods. Data collected by the DHIS for all patients with diabetes in KZN from 1 January 2010 to 31 December 2014 inclusive were analysed. Additional open-source databases were accessed to enable further exploration of the data collected.

Results. The study showed that the majority (38.7\%) of patients with diabetes on the public sector register were from the district of eThekwini. Positive correlations were found between the prevalence of diabetes, the mortality rate and the number of defaulters (patients with diabetes who did not return for regular treatment).

Conclusions. Provincial estimates of the prevalence of diabetes in this study were higher than the known national prevalence. This may be due to the large proportion of Indians in KZN, who have a genetic predisposition to diabetes mellitus. However, allowance must be made for possible inaccurate data collection at source with miscounting of individuals. This study supports the global trend of an association between diabetes and urbanisation and highlights the need for regular diabetes screening and education, particularly in the public healthcare domain. S Afr Med J 2016;106(4):389-393. DOI:10.7196/SAMJ.2016.v106i4.10143
\end{abstract}

Developing, low-income countries not only confront the challenge of resource constraints, an ageing population and urbanisation, but also face the double burden of communicable diseases such as HIV/ AIDS, which is currently the African continent's leading cause of death, and diabetes mellitus. ${ }^{[1]}$ According to global estimations, it is predicted that by the year 2030, developing countries will experience as much as a $69 \%$ increase in the number of adults diagnosed with diabetes. ${ }^{[2]}$ Diabetes caused more than 68 thousand deaths in South Africa (SA) in 2013. ${ }^{[3]}$ Census information for the year 2013 indicates that in KwaZulu-Natal (KZN), a subtropical province of SA with the country's second-largest population, diabetes was the fourth-largest cause of death. ${ }^{[4,5]}$

More than any other continent, Africa suffers from the combined presence and effects of diabetes together with HIV/AIDS and tuberculosis (TB). The interactions between these conditions and their treatment pose many challenges. Patients with diabetes have been found to have an increased general risk of infection and a two to three times increased risk of developing TB. HIV has been found to promote recurrence of latent $\mathrm{TB}$ and drastically increases the progression to active disease. ${ }^{[6]}$ Some antiretrovirals cause glucose intolerance, predisposing the HIV-positive patient to developing diabetes. Drug interactions between the medications used to treat diabetes and TB reduce each other's effectiveness, making it difficult to treat both conditions in one patient. ${ }^{[7]}$

The International Diabetes Federation's 2013 Diabetes Atlas ${ }^{[3]}$ indicates that $\mathrm{SA}$ is home to more than two and a half million diagnosed diabetics, and an approximate further million who are undiagnosed. It has been estimated that another 2.6 million South Africans have impaired glucose tolerance, an early metabolic abnormality that often leads to the eventual development of diabetes. $^{[3]}$ Throughout Africa there are approximately 20 million reported cases of diabetes, although $62 \%$ of patients remain undiagnosed. ${ }^{[3]}$ This suggests that a significant number of diabetics are at risk of developing chronic complications arising from abnormal blood glucose, and remain unaware of the treatment methods required to improve their prognosis until the manifestations are at an advanced stage. ${ }^{[1]}$

Documenting the assumed prevalence of diabetes based on healthcare access and noting the distribution of facilities at which individuals can access healthcare is important for a number of reasons. The findings may confirm previously described differences in prevalence based on the degree of urbanisation of the population, ${ }^{[8]}$ or may show an unexpected prevalence in certain districts, suggesting underdiagnosis or inaccurate assessment of the presence of risk factors for the development of diabetes. Describing the distribution of healthcare access will also enable more appropriate distribution of resources for the management of diagnosed patients, or may highlight the need for more intensive screening for possible undiagnosed cases of diabetes.

\section{Objective}

To determine the distribution, incidence, crude prevalence and prevalence of patients with diabetes mellitus in KZN and its districts during the 5-year period 2010 - 2014, as measured by the number of patients presenting at public health institutions. In addition, the distribution of patients who defaulted from treatment and the difference between the districts in terms of economic status was calculated. 


\section{Methods}

The District Health Information System (DHIS) is the primary data collection system of the Department of Health in KZN. Data are routinely collected at all public healthcare facilities in the province and aggregated per facility, with no individual patient identifiers. Data collected by the DHIS for all patients with diabetes seen at public healthcare facilities in KZN from 1 January 2010 to 31 December 2014 inclusive were obtained and analysed after ethics approval from the University of KwaZuluNatal ethics committee (HSS/0036/015M) and the KwaZulu-Natal Department of Health (HRKM 033/15).

The open-source database of the National Health Trust (NHT) was accessed for estimated medical scheme coverage. The South African Index of Multiple Deprivation $(\text { SAIMD })^{[9]}$ was accessed for data on socioeconomic status of the districts of KZN and data to assess the relationship between diabetes and urbanisation.

In 2013, additional data collection fields were added to the DHIS database to document the number of diabetes patients who had defaulted treatment. The distribution of these patients among the districts of KZN was analysed to determine the degree of non-compliance and assess its relationship with mortality as recorded by the diabetes mortality estimates reported by Statistics South Africa in 2013. ${ }^{[4]}$

Data were analysed using Microsoft Excel 2013, and pivot tables were generated to scrutinise the various and individual data points. Time series analyses conducted on several data categories provided insight into data trends and rates. Cross-sectional analyses were also conducted to understand the general dispersion of different variables, and to identify areas of high incidence as well as any anomalies requiring in-depth research. Graphical representation of the analyses was conducted using Think-Cell, version 6 software.

A normalised average would have been skewed with the inclusion of older data, so a true reflection of the current distribution of new cases of diabetes in KZN was drawn from the most recent 2 years (2013 and 2014). Data points closer to the start of the 5-year period could have contained extraneous factors that might have compromised the current distribution. Economic and social factors (e.g. urbanisation, employment trends and migration) could have changed fundamentally over the 5-year period, compromising the validity and robustness of the results. In order for policy recommendations to be properly formulated and deployed, the information presented needs to be the most recent reflection of the current state.

Data from the NHT were used to exclude private healthcare users and calculate the incidence of diabetes in the public sector. The total number of new patients recorded per district was divided by the public sector populations and then multiplied by 10000 to allow for the comparison of whole numbers. The resulting incidence rates were represented per 10000 patients.

Analysis of the prevalence rate over a 5 -year period would have been ambiguous, as incidence rates between the years 2010 and 2014 were calculated in the study. In essence, by determining the incidence rate, the growth of the condition between the years analysed would ideally equal the rate of change of prevalence over the same time period. Nevertheless, it would be interesting to calculate the prevalence of diabetes over the time period analysed, if only to compare the change in prevalence rate found with that of the incidence rate, to determine the accuracy of data collection and capturing. Medical scheme coverage data were used to exclude the population of private health users from the denominator in order to determine the prevalence of diabetes in the public sector.
Various studies have estimated the proportion of undiagnosed diabetic patients at between $30 \%$ and $70 \%$ of the known diabetic population, but there are few existing data that take race and age into account. Using data from the South African National Health and Nutrition Examination Survey (SANHANES), the undiagnosed diabetic population was calculated to be $48 \%^{[10]}$ and was used to calculate the estimated prevalence of diabetes.

\section{Results \\ Distribution}

A normalised 2-year average (2013/2014) was used to represent the true distribution of diabetes mellitus in KZN and then converted to percentages, as displayed in Fig. 1. The distribution ranged from 3\% (uMkhanyakude) to $38.7 \%$ (eThekwini).

\section{Incidence}

This study found that $\sim 50 \%$ of all patients newly diagnosed with diabetes mellitus from 2010 to 2014 were from the district of eThekwini. The incidence rate found in this study was expected to follow national and international trends of increasing annual incidence of diabetes; however, the results displayed a fluctuating incidence between 2010 and 2014.

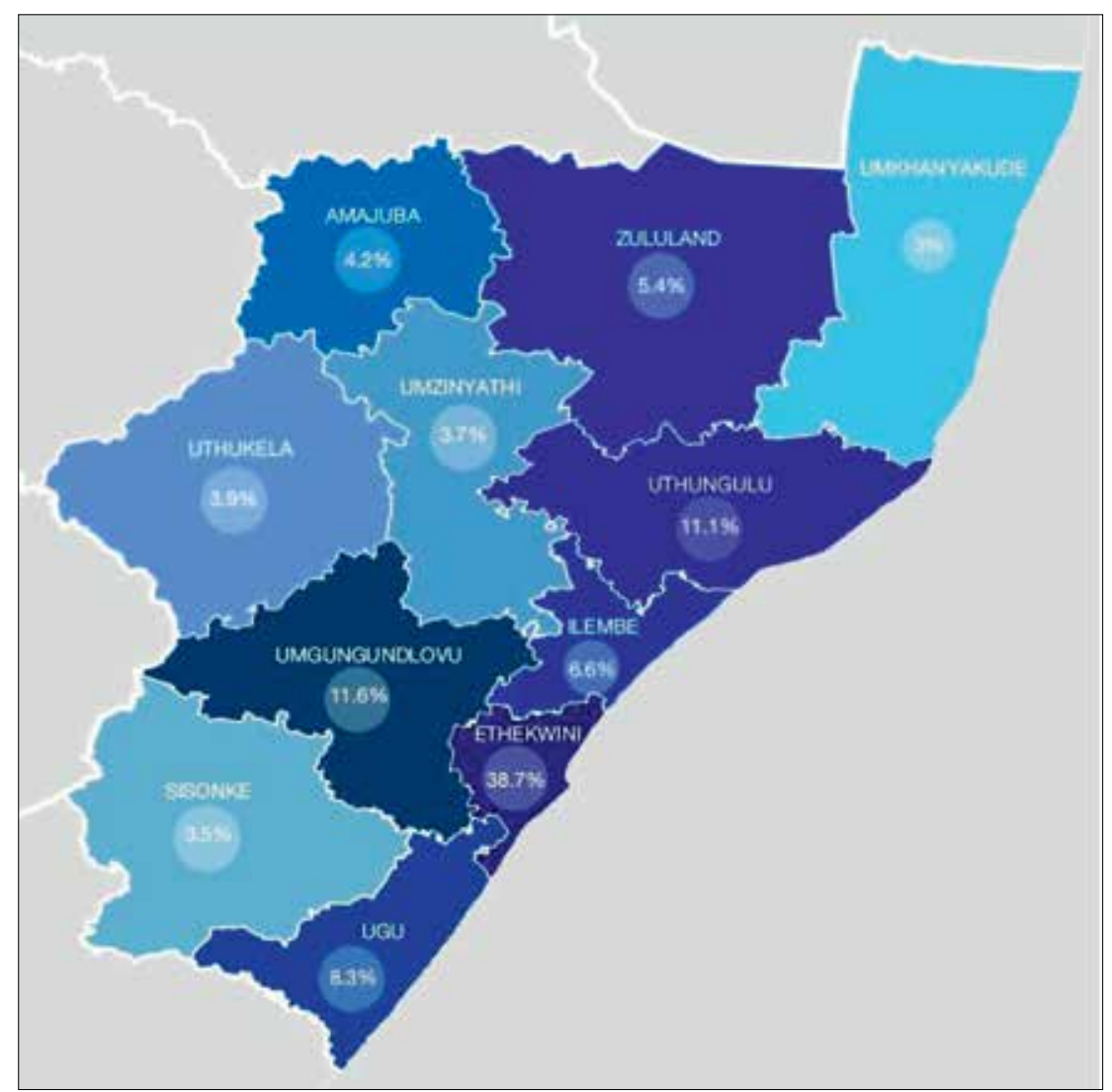

Fig. 1. Map showing the distribution of patients diagnosed with diabetes in the public sector in KZN. 
The fluctuation in the incidence rate can be observed from the increasing and decreasing percentage values found between the bars in Fig. 2, each of which represents a year of data that was analysed. A drastic reduction in the incidence rate can be observed between the years 2010 and 2011, mainly owing to a change in the numbers of new patients recorded in the district of Amajuba. A second drop in the incidence rate is seen between 2012 and 2013. Analysis of incidence by age, recorded in 2013 and 2014, revealed that in all districts in both these years the number of newly diagnosed diabetics aged $>19$ years was higher than that of patients aged $\leq 19$.

\section{Prevalence}

The crude prevalence of diabetes mellitus for KZN was calculated as $12.5 \%$ for the year 2014. Estimated medical scheme coverage for the province was $12.6 \%$. It was therefore calculated that the provincial prevalence of diabetes in KZN in patients accessing the public health sector was $14.3 \%$.

The estimated prevalence of diabetes in KZN of $34.1 \%$ implied that one in every three patients visiting a public health facility in the province had diabetes. This was factored into the estimated diabetes prevalence rates for KZN and the total diabetes prevalence rates for the districts (Figs 3 and 4). As seen in Fig. 4, the estimated diabetes prevalence per district was as high as $43.5 \%$ in the district of uThungulu.

\section{Defaulters}

Normalised averages revealed that the district of eThekwini had the highest record of defaulting patients. However, a fluctuation in the trend of the number of defaulters recorded for 2013 and 2014 was seen; six districts experienced a decrease in the number of defaulters between the 2 years, four appeared to have an increase, and one experienced no change. Fig. 5 presents

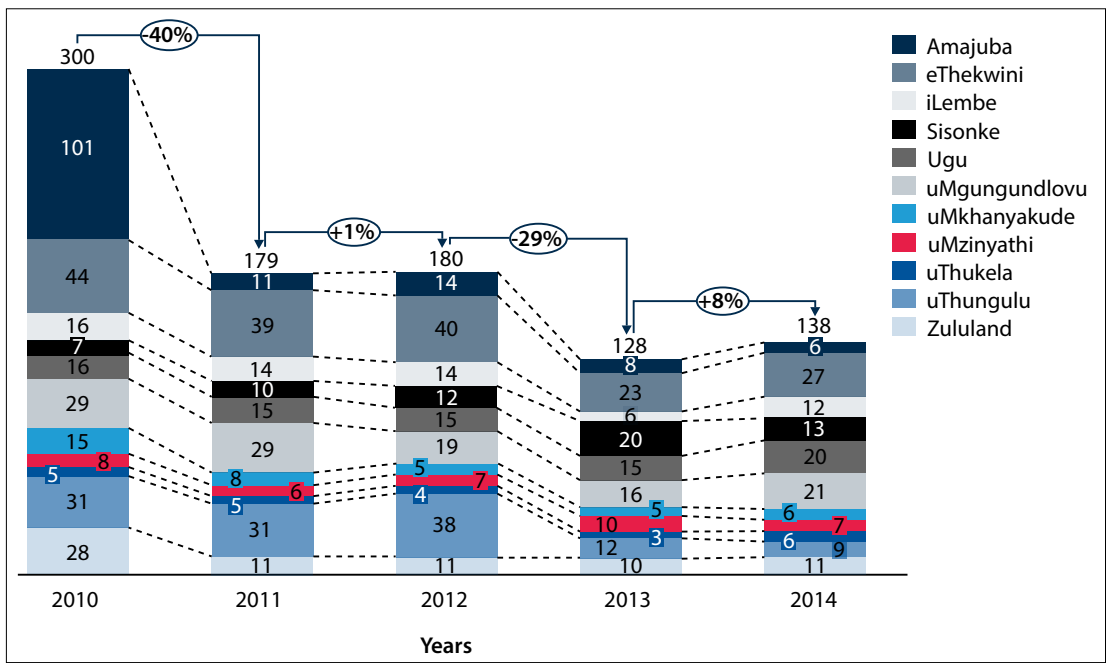

Fig. 2. Diabetes incidence rate timeline by district, 2010 - 2014 (/10 000 patients). the data collected on the distribution and number of defaulters, numbers ranging from 898 to over 10000 . Fig. 5 also indicates the percentage difference between 2013 and 2014 by district.

Correlation coefficients were used to represent the linear interdependence of two variables or sets of data. Individual correlation coefficients between each socioeconomic domain ${ }^{[9]}$ and the prevalence of diabetes in the public sector were calculated and are displayed in Table 1, which shows that all correlations between diabetes prevalence and the socioeconomic domains yielded negative coefficients. The diabetes mortality rates for the districts of KZN for the year $2013^{[4]}$ were correlated with the diabetes defaulters recorded for that year and resulted in a correlation coefficient of 0.85 .

\section{Discussion}

The incidence of diabetes mellitus in KZN fell by $56 \%$ between 2010 and 2014. This figure is not consistent with the literature, as many sources report a growing incidence

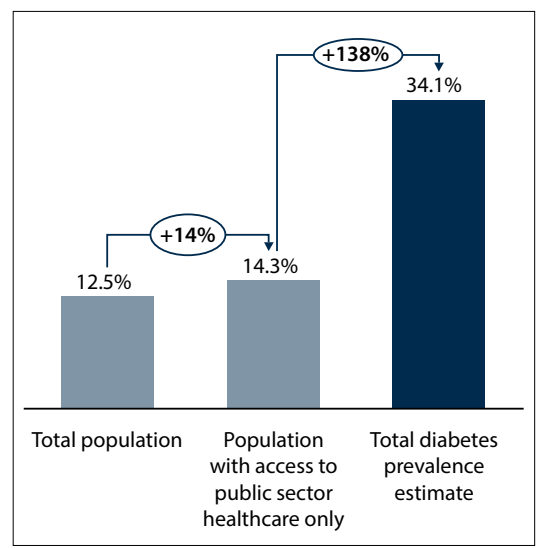

Fig. 3. Diabetes prevalence rates (\%) in KZN.

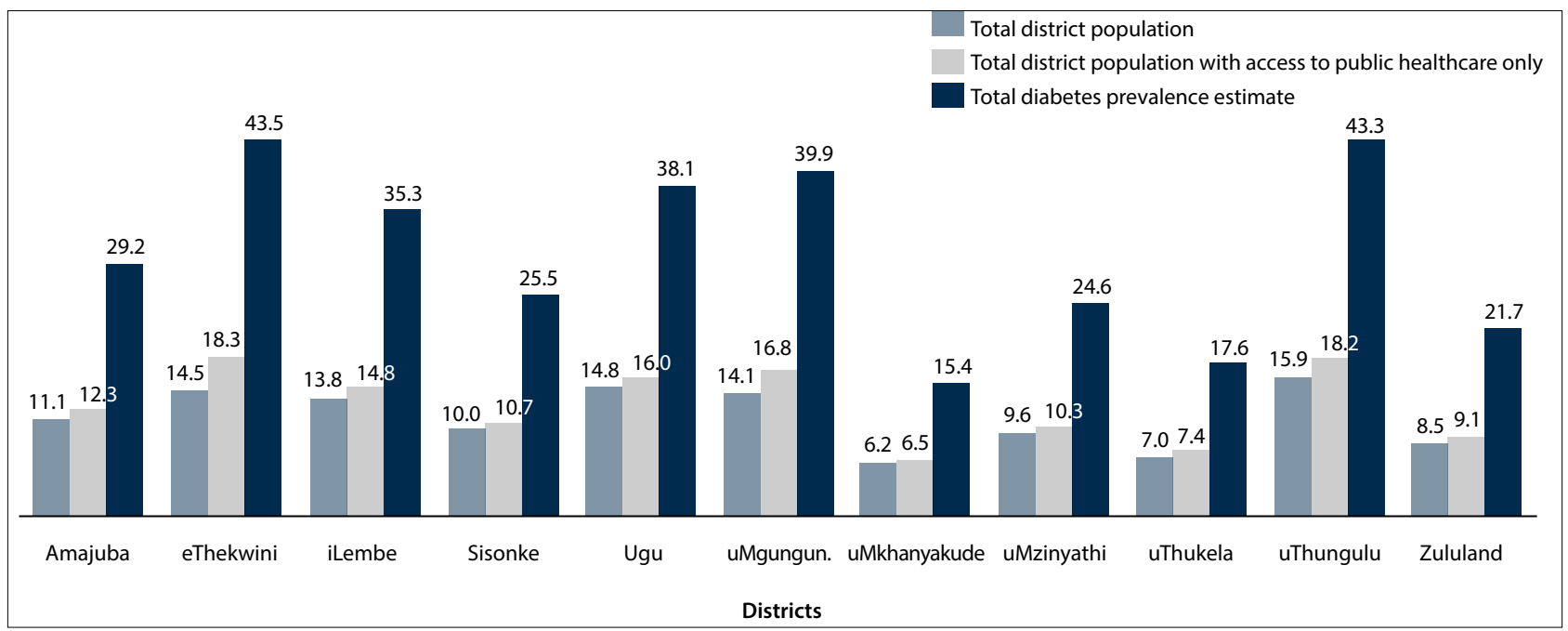

Fig. 4. Total estimated diabetes prevalence (\%) for the districts of KZN. 


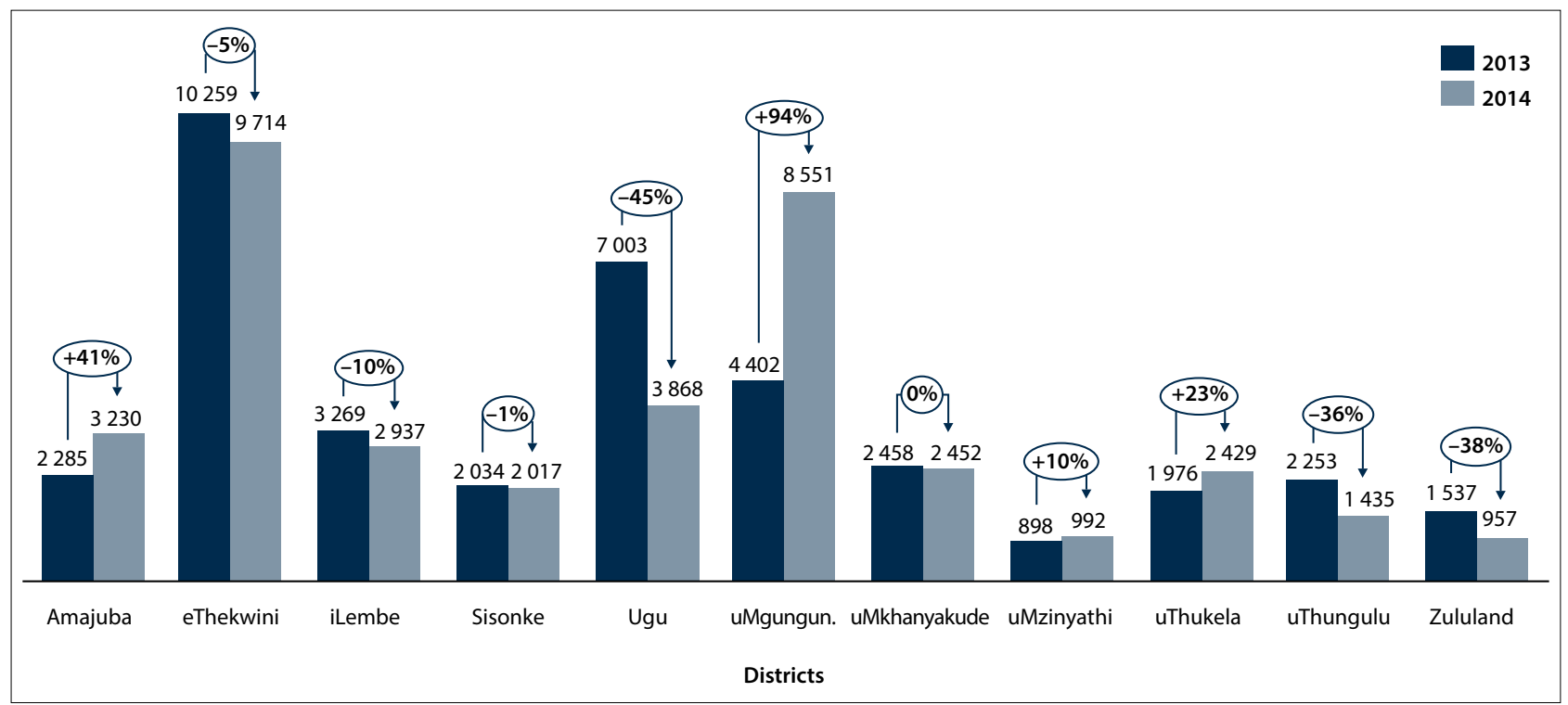

Fig. 5. Distribution of defaulting diabetic patients $(\mathrm{n})$ in the districts of KZN.

Table 1. Correlation coefficients of diabetes prevalence and socioeconomic domains

\begin{tabular}{lll}
\hline Correlation & Value & Result \\
\hline Prevalence and income & -0.54 & A moderate negative relationship \\
Prevalence and employment & -0.14 & A weak negative linear relationship \\
Prevalence and education & -0.45 & A moderate negative relationship \\
Prevalence and living environment & -0.41 & A weak-moderate negative relationship
\end{tabular}

and prevalence of diabetes, especially on the African continent. ${ }^{[3]}$ The fluctuation in the number of new patients diagnosed with diabetes could be attributed to inconsistent recording, possible capture errors and the misunderstanding of additional data collection fields.

With regard to the incidence of diabetes by age profile, the results were consistent with the existing literature, indicating that the incidence and prevalence of diabetes in individuals aged $>19$ years is far greater than in those $\leq 19$ years of age. ${ }^{[11]}$ Data collection does not differentiate between the different types of diabetes, but it is safe to assume that the majority of older patients are type 2 diabetics. The distribution of diabetes by age in the districts of eThekwini, iLembe, uMgungundlovu, uMkhanyakude and uThukela showed that of patients with diabetes in these districts, $>10 \%$ were aged $<18$ years, suggesting that the number of patients aged $>19$ is grossly underreported (assuming that the expected ratio of type 1 and 2 diabetics is valid in these populations and that most of the younger patients have type 1 diabetes $\left.{ }^{[11]}\right)$. According to the study, $44-54 \%$ of the total number of newly diagnosed diabetics for each year was recorded in the district of eThekwini.
This study found that the crude prevalence of diabetes for KZN was $12.5 \%$, which is higher than the known national prevalence of $9.2 \% .{ }^{[2]}$ This may be attributed to the large population of Indians in $\mathrm{KZN},{ }^{[5]}$ as Indians have been reported to have a genetic predisposition to type 2 diabetes. ${ }^{[12]}$ Our findings are consistent with other epidemiological studies in SA that reported a prevalence of $>12 \%$ in the Indian population. ${ }^{[13]}$ The KZN prevalence rate in the public sector was $14.3 \%$. The increase was expected, as the denominator was reduced owing to exclusion of the private health sector population.

Early detection of diabetes enables early intervention and the prevention of damaging and costly complications. The population of undiagnosed patients is thought to be high in African countries, where the minimum standard of diabetes care and screening is difficult to achieve. ${ }^{[3]}$ Once the undiagnosed percentage of diabetic patients as found in the SANHANES study ${ }^{[10]}$ was factored into the prevalence calculations done for this study, a diabetes prevalence of $34.1 \%$ was estimated for KZN. This implies that more than a third of patients seen at public healthcare facilities may have diabetes. An estimated diabetes prevalence of such magnitude further emphasises the urgent need for education, prevention initiatives, screening protocols, better management of the disease and its complications, the establishment of multidisciplinary teams, and an improved data collection system that will enable accurate measurement of disease progression.

The top five high-risk districts (eThekwini, uThungulu, uMgungundlovu, Ugu and iLembe) were the only districts calculated to have an estimated total prevalence of diabetes above the estimated provincial total prevalence of $34.1 \%$. eThekwini had the highest estimated total diabetes prevalence at $43.5 \%$. Of the 11 districts of KZN, eThekwini is the most densely populated, is the only district to fall within the 5th (highest) socioeconomic quintile, and is highly urbanised. ${ }^{[14]}$ In addition to the large Indian population (who, as mentioned above, have a predisposition to diabetes), eThekwini is home to a vast population of individuals who subscribe to the traditional African belief that obesity is a symbol of affluence and good health. ${ }^{[5,12]}$

All correlation coefficients of the deprivation domains and prevalence calculations yielded a negative result; hence, the more deprived or less urbanised an area, the lower the prevalence of diabetes.

Urbanisation, i.e. movement of the population into urban areas, has been thought to improve the standard of living by improving access to public healthcare, transport, stable housing and clean drinking water, but is not without its disadvantages. In developing countries such as SA, the infrastructure is often inadequate to support densely populated urban areas so there is an increase 
in environmental pollution and unemployment and the development of slums. According to a report released by the Department of Economic Development and Tourism in 2010, eThekwini is 45\% urbanised and the rate is steadily increasing. ${ }^{[15]}$

Along with the abovementioned disadvantages of urbanisation come a change in lifestyle and a consequent increase in the risk of type 2 diabetes. Factors such as infrequent physical activity owing to the use of motor vehicles as opposed to walking, and a change in diet to cheaper, more processed fast foods, are known to increase the risk of diabetes ${ }^{[8]}$ The categorisation of eThekwini as a high-priority district is further justified by the prevalence calculations highlighted in this study. The distribution of defaulting patients follows a similar trend, as the number of defaulting patients recorded was highest in the district of eThekwini.

The correlation coefficient of 0.85 between the diabetes mortality rate and the number of defaulting patients recorded for the same year infers a very strong positive relationship between the two variables. These findings support the theory that poor compliance with diabetes treatment leads to a poor prognosis. Alternatively, patients who died may have been reported as defaulters. This needs to be explored further in future studies.

\section{Conclusion}

With regard to the distribution of diabetes mellitus, the study findings indicate that the majority of diabetes patients on the public sector register (38.7\%) were from the district of eThekwini. Similar results were found for calculations on incidence and defaulters, indicating that, with the exception of the incidence in the year 2010, the greatest incidence and largest proportion of defaulters occurred in eThekwini, while provincial calculations fluctuated between the years investigated. The provincial crude prevalence of diabetes was found to be $12.5 \%$, increasing to $34.1 \%$ when members of private medical aids were excluded and an estimation of undiagnosed patients was factored into the prevalence calculations. Prevalence calculations by district revealed that the five districts with the highest prevalence calculations had readings above the provincial prevalence estimation. The trends identified by this study support the recurring global issue of the relationship between diabetes and urbanisation. It is also possible that districts with a higher deprivation score are illequipped to screen for diabetes and to record cases, and that residents of low socioeconomic status lack education on the importance of screening, resulting in a relatively low number of patients being recorded as having diabetes.

In conclusion, this article highlights the need for and importance of diabetes education, prevention initiatives, screening protocols, better management of the disease and its complications, the establishment of multidisciplinary teams and an improved data collection system particularly in the high-risk areas identified in this study.

Author contributions. NS researched the data, conducted the data analysis and prepared the first draft of the manuscript. VSS and SB reviewed/edited and contributed to the introduction, methods and discussion.

Acknowledgements. We thank the KZN Department of Health for access to the data. This publication was made possible by grant number R24TW008863 from the Office of the US Global AIDS Coordinator and the US Department of Health and Human Services, National Institutes of Health (NIH OAR and NIH ORWH). Its contents are solely the responsibility of the authors and do not necessarily represent the official views of the government.

\section{References}

1. Mbanya JC, Motala AA, Sobngwi E, Assah FK, Enoru ST. Diabetes in sub-Saharan Africa. Lancet 2010;375(9733):2254-2266. DOI:10.1016/S0140-6736(10)60550-8

2. Shaw JE, Sicree RA, Zimmet PZ. Global estimates of the prevalence of diabetes for 2010 and 2. Shaw JE, Sicree RA, Zimmet PZ. Global estimates of the prevalence of diabers
2030. Diabetes Res Clin Pract 2010;87(1):4-14. DOI:10.1016/j.diabres.2009.10.007

2030. Diabetes Res Clin Pract 2010;87(1):4-14. DOI:10.1016/j.diabres.2009.10.007
3. International Diabetes Federation. IDF Diabetes Atlas. 6th ed. 2013. www.idf.org/diabetesatlas (accessed 10 March 2015)

4. Statistics South Africa. Mortality and causes of death in South Africa, 2013: Findings from death notification, 2014. http://www.statssa.gov.za/publications/P03093/P030932013.pdf (accessed 20 April 2015)

5. Statistics South Africa. Census 2011. Pretoria: SSA, 2012

6. Harries AD, Lin Y, Satyanarayana S, et al. The looming epidemic of diabetes-associated tuberculosis: Learning lessons from HIV-associated tuberculosis. Int J Tuberc Lung Dis 2011;15(11):1436-1444 DOI: $10.5588 /$ iitld. 11.0503

7. Health24. Diabetes, TB and HIV/Aids, 2010. http://www.health24.com/Medical/Diabetes/Who-is-atrisk/Diabetes-TB-and-HIVAids-20120721 (accessed 15 April 2015).

8. Akter S, Rahman MM, Abe SK, Sultana P. Prevalence of diabetes and prediabetes and their risk factors among Bangladeshi adults: A nationwide survey. Bull World Health Organ 2014;92(3):204-213. DOI: 10.2471/BLT.13.128371

9. Wright G, Noble M. South African Department of Social Development - the South African Index of Multiple Deprivation 2007 at Municipality Level. 2007. http://www.casas.ox.ac.uk/imd.html of Multiple Deprivation
(accessed 5 March 2015).

10. Human Sciences Research Council. SANHANES - launch edition, 2013. http://www.hsrc.ac.za/ uploads/pageNews/72/SANHANES-launch\%20edition\%20\%28online\%20version\%29.pdf (accessed 15 April 2015).

11. Healthline. Type 2 diabetes statistics and facts, 2014. http://www.healthline.com/health/type-2diabetes/statistics (accessed 15 April 2015).

12. Omar MA, Seedat MA, Dyer RB, et al. South African Indians show a high prevalence of NIDDM and bimodality in plasma glucose distribution patterns. Diabetes Care 1994;17(1):70-73. DOI:10.2337 diacare.17.1.70

13. Dikeukwu RA. The awareness and practices of foot self-care in patients with diabetes at Dr Yusuf Dadoo district hospital, Johannesburg. Unpublished master's thesis. Johannesburg: University of Witwatersrand, 2011.

14. Health Systems Trust. District Health Barometer 2013/14. Durban: HST, 2014. http://www.hst.org.za/ publications/district-health-barometer-201314 (accessed 15 April 2015).

15. Beires L. KwaZulu-Natal Department of Economic Development and Tourism - a Study of Urbanisation in the Province of KwaZulu-Natal. 2010. http://www.kznded.gov.za/Portals/0/ OUTLINE\%20OF\%20URBANISATION\%20DEDT.pdf (accessed 20 April 2015).

Accepted 14 October 2015. 